\title{
2007 Agricultural Census Tidbit: Dominant Farm Organizational Structure in Florida Is Small Family Farms ${ }^{1}$
}

Rodney L. Clouser ${ }^{2}$

\section{Introduction}

The 2007 agricultural census data were reported for states and counties throughout the United States in February 2009. National, state, and local policy makers look forward to the census report for several reasons: they use the information to show the importance and size of the industry in their state and counties, and to show the importance of the industry in the state and local economy; and they use the information to make policy decisions. One of the limitations of the agricultural census is that the report, for the most part, is just data with limited or no discussion. This publication is one in a series of five that reorganizes some of the data collected in the census and offers some comments on trends and gaps in the data. The other publications in the 2007 Agricultural Census Tidbit series can be accessed online at http://edis.ifas.ufl.edu.

Many Floridians, including those not directly involved in farm production, keep a close watch on the numbers and types of farms in the state. People's interest in this topic is varied, but Floridians who are not farmers may be concerned that big farms and non-family farms represent an increasing percentage of farms in the state, and they may consider this trend to be undesirable.

\section{Census of Agriculture}

The Census of Agriculture is conducted every five years and is an attempt by the U.S. government to collect data on the entire U.S farm population in every state. Of course, not all agricultural producers provide the information requested and there is always the possibility for error in the data reported. A farm is defined as a place in which $\$ 1,000$ of agricultural products were produced or sold, or typically would have been sold during the census year, including any government payments.

Data in this publication are for 2007 only. While the census has been collecting data on the number of farms by size for years, in 2007 they began collecting information on farm typology. This was done so that farms could be grouped by similar characteristics. In total, eight different categories were tabulated: five under small farms and three under other farms. Typology characteristics are summarized in Table 1.

1. This is EDIS document FE806, a publication of the Food and Resource Economics Department, Florida Cooperative Extension Service, Institute of Food and Agricultural Sciences, University of Florida, Gainesville, FL. Published November 2009. Please visit the EDIS website at http://edis.ifas.ufl.edu.

2. Rodney L. Clouser, professor and extension public policy specialist, Food and Resource Economics Department, Florida Cooperative Extension Service, Institute of Food and Agricultural Sciences, University of Florida, Gainesville, FL.

The Institute of Food and Agricultural Sciences (IFAS) is an Equal Opportunity Institution authorized to provide research, educational information and other services only to individuals and institutions that function with non-discrimination with respect to race, creed, color, religion, age, disability, sex, sexual orientation, marital status, national origin, political opinions or affiliations. U.S. Department of Agriculture, Cooperative Extension Service, University of Florida, IFAS, Florida A. \& M. University Cooperative Extension Program, and Boards of County Commissioners Cooperating. Millie FerrerChancy, Interim Dean 


\section{Farm Size and Typology}

In 2007 as in the past, Florida data indicated that the primary and dominant organizational structure in Florida agriculture is small farms. The number of farms by acreage categories is reported in Table 2 . While the census collects information on farm size in 12 different categories, for this publication the 12 have been combined and reduced to four categories: 1 to 9.9 acres, 10 to 49.9 acres, 50 to 999 acres, and greater than 1,000 acres.

Florida has more than 47,400 farms and of these farms, just over 69 percent are 50 acres or less in size. This ranks Florida as the state with the 4th largest percentage of farms of less than 50 acres. There are several reasons for the relatively small acreage of Florida farms that will be discussed under farm typology, but one obvious reason for relatively small farms is the high-value, intensive nature of commodities produced in Florida. Many sales and much income can be generated from horticulture operations, specialty crops, and some fruit and vegetable crops.

In contrast, Florida only has 2.9 percent of farms greater than 1,000 acres. This places the state in the bottom 20 for farms greater than 1,000 acres. The 2007 census reported the number of farm operations greater than 1,000 acres down, from 1,769 in 2002 to 1,370 in 2007 (a decrease of about 23\%). Likewise, farms less than 50 acres increased to over 32,800 in 2007, down from over 28,600 in 2002 (an increase of about $15 \%$ ). Florida's organizational structure is dominated by small farms, but the acreage in these operations is relatively small. While farms less than 50 acres accounted for 69 percent of farms, acreage in the operations accounted for only five percent of land in farms in 2007. Farms larger than 1,000 acres represent only 2.9 percent of farms in the state but account for 66 percent of land in farms in 2007.

Florida farm typology numbers (Table 3 ) reveal that the largest number of small farms can be categorized as retirement farms (sales less than $\$ 250,000$, with the primary occupation of the landowner reported as "retired"), and residential/lifestyle (sales less than $\$ 250,000$, with the landowner's primary occupation reported as something other than farming). These two categories account for about 60 percent of all small farms in the state. On the opposite end of the scale, only 1.7 percent of farms are considered large (sales between $\$ 250,000$ and $\$ 499,999$ ) and 3.1 percent are categorized as very large (sales greater than $\$ 500,000$ ), and only 5.9 percent are structured as nonfamily corporations or as operated by hired managers.

Retirement and residential/lifestyle farms were further reviewed since they are the two largest farm categories in Florida. Data indicated that there are similarities as well as differences when comparing Florida to other southern states (Table 4). For example, 11 of the top 15 states in percentage of farms categorized as retirement were southern states. In terms of residential/lifestyle farms, 11 southern states have a larger percentage of farms identified in this typology category than does Florida. The census does not collect information documenting how individual operators ended up in the typology categories. Local culture, the availability of off-farm employment, or the desire of newcomers to the state to recreate a farming lifestyle like that they remember from their old homes might all be factors influencing retirement and residential/lifestyle typology.

\section{Summary}

The dominant organizational structure of agriculture in Florida is individual family farms. Just over 69 percent of Florida agricultural operations are less than 50 acres in size. In terms of farm typology in Florida, the two dominant categories are retirement (23.5\%) and residential/lifestyle (34.9\%). Together, these two categories account for almost six of every ten agricultural operations in Florida.

\section{References}

USDA/NASS. 2007. 2007 Census of Agriculture, United States Summary and State Data, Volume 1, Geographic Area Series, Part 51. United States Department of Agriculture, National Agricultural Statistics Service, Washington, D.C.

USDA/NASS. 2009. General Explanation and Census of Agriculture Report Form, Appendix B. 
United States Department of Agriculture, National

Agricultural Statistics Service, Washington, D.C. 
Table 1. Farm typology definitions.

\begin{tabular}{|llll|}
\hline \hline Type & Category & Sales Conditions & Other Conditions \\
\hline Small & Limited-resource & $<\$ 100,000$ & $<\$ 20,000$ in income \\
& Retirement farms & $<\$ 250,000$ & Operator retired \\
& Residential / lifestyle & $<\$ 250,000$ & $\begin{array}{l}\text { Primary occupation reported is something } \\
\text { other than farming }\end{array}$ \\
& Lower-sales farms & $<\$ 100,000$ & Primary occupation reported is farming \\
& Higher-sales farms & $\$ 100,000$ to $\$ 249,999$ & Primary occupation reported is farming \\
\hline Other & Large family farms & $\$ 250,000$ to $\$ 499,999$ & \\
& Very large family farms & $>\$ 500,000$ & \\
& Nonfamily farms & & $\begin{array}{l}\text { Organized as nonfamily corporations, or } \\
\text { operated by hired managers }\end{array}$ \\
& & & \\
\hline \hline
\end{tabular}

Table 2. Farm operation by size, ranked by 50 acres or less, 2007.

\begin{tabular}{|c|c|c|c|c|c|c|c|}
\hline \multirow[t]{2}{*}{ State } & $\begin{array}{r}1.0 \text { to } 9.9 \\
\text { Acres }\end{array}$ & $\begin{array}{r}10.0 \text { to } 49.9 \\
\text { Acres }\end{array}$ & $\begin{array}{r}50 \text { to } 999.9 \\
\text { Acres }\end{array}$ & $\begin{array}{r}>1,000 \\
\text { Acres }\end{array}$ & $\begin{array}{c}\text { Total } \\
\text { Farms }\end{array}$ & $\begin{array}{l}<50 \\
\text { Acres }\end{array}$ & $\begin{array}{r}>1,000 \\
\text { Acres }\end{array}$ \\
\hline & (Value) & (Value) & (Value) & (Value) & (Value) & (\%) & (\%) \\
\hline Hawaii & 4,813 & 1,972 & 621 & 115 & 7,521 & 90.2 & 1.5 \\
\hline Arizona & 9,873 & 2,657 & 2,283 & 824 & 15,637 & 80.1 & 5.3 \\
\hline New Jersey & 2,950 & 4,814 & 2,455 & 108 & 10,327 & 75.2 & 1.0 \\
\hline Florida & 12,184 & 20,680 & 13,229 & 1,370 & 47,463 & 69.2 & 2.9 \\
\hline Rhode Island & 353 & 484 & 380 & 2 & 1,219 & 68.7 & 0.2 \\
\hline Massachusetts & 2,199 & 2,885 & 2,579 & 28 & 7,691 & 66.1 & 0.4 \\
\hline California & 25,278 & 28,080 & 23,220 & 4,455 & 81,033 & 65.8 & 5.5 \\
\hline Connecticut & 1,232 & 1,894 & 1,750 & 40 & 4,916 & 63.6 & 0.8 \\
\hline Oregon & 9,546 & 14,142 & 12,301 & 2,564 & 38,553 & 61.4 & 6.7 \\
\hline Washington & 9,211 & 14,790 & 12,517 & 2,766 & 39,284 & 61.1 & 7.0 \\
\hline Delaware & 651 & 802 & 947 & 146 & 2,546 & 57.1 & 5.7 \\
\hline Utah & 4,194 & 5,127 & 6,073 & 1,306 & 16,700 & 55.8 & 7.8 \\
\hline
\end{tabular}


Table 2. Farm operation by size, ranked by 50 acres or less, 2007.

\begin{tabular}{|c|c|c|c|c|c|c|c|}
\hline State & $\begin{array}{r}1.0 \text { to } 9.9 \\
\text { Acres }\end{array}$ & $\begin{array}{r}10.0 \text { to } 49.9 \\
\text { Acres }\end{array}$ & $\begin{array}{r}50 \text { to } 999.9 \\
\text { Acres }\end{array}$ & $\begin{array}{r}>1,000 \\
\text { Acres }\end{array}$ & $\begin{array}{c}\text { Total } \\
\text { Farms }\end{array}$ & $\begin{array}{l}<50 \\
\text { Acres }\end{array}$ & $\begin{array}{r}>1,000 \\
\text { Acres }\end{array}$ \\
\hline & (Value) & (Value) & (Value) & (Value) & (Value) & $(\%)$ & (\%) \\
\hline New Mexico & 6,471 & 4,405 & 6,499 & 3,555 & 20,930 & 52.0 & 17.0 \\
\hline New Hampshire & 754 & 1,405 & 1,969 & 38 & 4,166 & 51.8 & 0.9 \\
\hline Idaho & 4,891 & 7,497 & 10,478 & 2,483 & 25,349 & 48.9 & 9.8 \\
\hline Nevada & 631 & 898 & 1,155 & 447 & 3,131 & 48.8 & 14.3 \\
\hline North Carolina & 5,001 & 20,772 & 25,478 & 1,662 & 52,913 & 48.7 & 3.1 \\
\hline Indiana & 9,720 & 19,533 & 27,779 & 3,906 & 60,938 & 48.0 & 6.4 \\
\hline Alaska & 165 & 164 & 305 & 52 & 686 & 48.0 & 7.6 \\
\hline Maryland & 1,554 & 4,589 & 6,325 & 366 & 12,834 & 47.9 & 2.9 \\
\hline Louisiana & 3,282 & 10,394 & 14,508 & 1,922 & 30,106 & 45.4 & 6.4 \\
\hline Michigan & 4,219 & 20,726 & 29,100 & 1,969 & 56,014 & 44.5 & 3.5 \\
\hline Tennessee & 5,814 & 29,396 & 42,722 & 1,348 & 79,280 & 44.4 & 1.7 \\
\hline Ohio & 7,767 & 24,361 & 41,019 & 2,714 & 75,861 & 42.4 & 3.6 \\
\hline South Carolina & 1,970 & 8,959 & 14,073 & 865 & 25,867 & 42.3 & 3.3 \\
\hline Maine & 1,046 & 2,383 & 4,527 & 180 & 8,136 & 42.1 & 2.2 \\
\hline Georgia & 3,504 & 16,243 & 26,146 & 1,953 & 47,846 & 41.3 & 4.1 \\
\hline Pennsylvania & 5,601 & 20,267 & 36,664 & 631 & 63,163 & 41.0 & 1.0 \\
\hline Alabama & 3,374 & 16,215 & 27,655 & 1,509 & 48,753 & 40.2 & 3.1 \\
\hline Virginia & 3,530 & 15,177 & 27,351 & 1,325 & 47,383 & 39.5 & 2.8 \\
\hline Illinois & 8,603 & 20,592 & 39,835 & 7,830 & 76,860 & 38.0 & 10.2 \\
\hline Texas & 21,024 & 72,837 & 142,250 & 11,326 & 247,437 & 37.9 & 4.6 \\
\hline Colorado & 4,276 & 9,359 & 17,162 & 6,257 & 37,054 & 36.8 & 16.9 \\
\hline Arkansas & 2,581 & 15,119 & 28,465 & 3,181 & 49,346 & 35.9 & 6.4 \\
\hline Vermont & 635 & 1,862 & 4,343 & 144 & 6,984 & 35.8 & 2.1 \\
\hline Kentucky & 4,579 & 25,235 & 53,701 & 1,745 & 85,260 & 35.0 & 2.0 \\
\hline New York & 2,914 & 8,799 & 23,600 & 1,039 & 36,352 & 32.2 & 2.9 \\
\hline Wisconsin & 4,861 & 19,895 & 51,751 & 1,956 & 78,463 & 31.6 & 2.5 \\
\hline West Virginia & 1,207 & 5,749 & 16,328 & 334 & 23,618 & 29.5 & 1.4 \\
\hline
\end{tabular}


Table 2. Farm operation by size, ranked by 50 acres or less, 2007.

\begin{tabular}{|c|c|c|c|c|c|c|c|}
\hline \multirow[t]{2}{*}{ State } & $\begin{array}{r}1.0 \text { to } 9.9 \\
\text { Acres } \\
\end{array}$ & $\begin{array}{r}10.0 \text { to } 49.9 \\
\text { Acres }\end{array}$ & $\begin{array}{r}50 \text { to } 999.9 \\
\text { Acres }\end{array}$ & $\begin{array}{r}>1,000 \\
\text { Acres }\end{array}$ & $\begin{array}{c}\text { Total } \\
\text { Farms } \\
\end{array}$ & $\begin{array}{l}<50 \\
\text { Acres }\end{array}$ & $\begin{array}{r}>1,000 \\
\text { Acres } \\
\end{array}$ \\
\hline & (Value) & (Value) & (Value) & (Value) & (Value) & $(\%)$ & (\%) \\
\hline Mississippi & 2,056 & 10,234 & 27,425 & 2,244 & 41,959 & 29.3 & 5.3 \\
\hline lowa & 8,709 & 17,824 & 58,872 & 7,451 & 92,856 & 28.6 & 8.0 \\
\hline Missouri & 3,868 & 25,186 & 72,963 & 5,808 & 107,825 & 26.9 & 5.4 \\
\hline Oklahoma & 3,802 & 18,700 & 56,343 & 7,720 & 86,565 & 26.0 & 8.9 \\
\hline Minnesota & 3,687 & 16,927 & 54,190 & 6,188 & 80,992 & 25.5 & 7.6 \\
\hline Montana & 1,917 & 5,462 & 12,354 & 9,791 & 29,524 & 25.0 & 33.2 \\
\hline Wyoming & 652 & 2,004 & 5,280 & 3,133 & 11,069 & 24.0 & 28.3 \\
\hline Kansas & 2,123 & 10,041 & 41,032 & 12,335 & 65,531 & 18.6 & 18.8 \\
\hline Nebraska & 2,270 & 6,581 & 27,659 & 11,202 & 47,712 & 18.6 & 23.5 \\
\hline South Dakota & 920 & 3,898 & 16,497 & 9,854 & 31,169 & 15.5 & 31.6 \\
\hline North Dakota & 387 & 2,268 & 17,465 & 11,850 & 31,970 & 8.3 & 37.1 \\
\hline
\end{tabular}

Table 3. Florida farms by typology, 2007.

\begin{tabular}{|llrr|}
\hline \hline Type & Category & Number of Farms & \% of Florida Farms \\
\hline Small & & 7,798 & 16.4 \\
& Limited-resource & 11,144 & 23.5 \\
& Retirement farms & 16,561 & 34.9 \\
& Residential / lifestyle & 6,019 & 12.7 \\
& Lower-sales farms & 877 & 1.8 \\
& Higher-sales farms & 821 & 1.7 \\
& & 1,455 & 3.1 \\
Other & Large family farms & 2,788 & 5,9 \\
& Very large family farms & & \\
& Nonfamily farms & & \\
& & & \\
& &
\end{tabular}


Table 4. Farm typology for retirement and residential / lifestyle categories, ranked by state, 2007.

\begin{tabular}{|c|c|c|c|c|c|c|c|}
\hline \multirow[t]{2}{*}{ Rank } & \multirow[t]{2}{*}{ State } & \multicolumn{2}{|c|}{ Retirement } & \multirow[t]{2}{*}{ Rank } & \multirow[t]{2}{*}{ State } & \multicolumn{2}{|c|}{ Residential / Lifestyle } \\
\hline & & (Number) & (\%) & & & (Number) & (\%) \\
\hline 1 & West Virginia & 6,420 & 27.2 & 1 & Utah & 6,986 & 41.8 \\
\hline 2 & Texas & 64,462 & 26.1 & 2 & Colorado & 15,498 & 41.8 \\
\hline 3 & South Carolina & 6,561 & 25.4 & 3 & Tennessee & 32,824 & 41.4 \\
\hline 4 & Alabama & 12,350 & 25.3 & 4 & Indiana & 24,744 & 40.6 \\
\hline 5 & Virginia & 11,967 & 25.3 & 5 & Oklahoma & 35,061 & 40.5 \\
\hline 6 & Mississippi & 10,357 & 24.7 & 6 & Texas & 99,316 & 40.1 \\
\hline 7 & Tennessee & 19,385 & 24.5 & 7 & Ohio & 30,434 & 40.1 \\
\hline 8 & Georgia & 11,367 & 23.8 & 8 & Missouri & 42,987 & 39.9 \\
\hline 9 & Oregon & 9,126 & 23.7 & 9 & Kentucky & 33,938 & 39.8 \\
\hline 10 & Oklahoma & 20,447 & 23.6 & 10 & Alabama & 18,948 & 38.9 \\
\hline 11 & Florida & 11,144 & 23.5 & 11 & New Jersey & 3,987 & 38.6 \\
\hline 12 & Kentucky & 19,921 & 23.4 & 12 & West Virginia & 9,079 & 38.4 \\
\hline 13 & Hawaii & 1,744 & 23.2 & 13 & Louisiana & 11,473 & 38.1 \\
\hline 14 & Louisiana & 6,923 & 23.0 & 14 & Mississippi & 15,960 & 38.0 \\
\hline 15 & New Mexico & 4,732 & 22.6 & 15 & South Carolina & 9,824 & 38.0 \\
\hline 16 & Michigan & 12,410 & 22.2 & 16 & Virginia & 17,759 & 37.5 \\
\hline 17 & North Carolina & 11,712 & 22.1 & 17 & Idaho & 9,494 & 37.5 \\
\hline 18 & Washington & 8,571 & 21.8 & 18 & Arkansas & 18,434 & 37.4 \\
\hline 19 & Missouri & 23,491 & 21.8 & 19 & Wisconsin & 28,860 & 36.8 \\
\hline 20 & California & 17,209 & 21.2 & 20 & Georgia & 17,514 & 36.6 \\
\hline 21 & Utah & 3,536 & 21.2 & 21 & Connecticut & 1,795 & 36.5 \\
\hline 22 & New Jersey & 2,124 & 20.6 & 22 & Michigan & 20,279 & 36.2 \\
\hline 23 & Arkansas & 9,932 & 20.1 & 23 & Oregon & 13,807 & 35.8 \\
\hline 24 & Maryland & 2,566 & 20.0 & 24 & Washington & 14,066 & 35.8 \\
\hline 25 & Ohio & 15,071 & 19.9 & 25 & Pennsylvania & 22,563 & 35.7 \\
\hline 26 & Connecticut & 960 & 19.5 & 26 & Maine & 2,884 & 35.4 \\
\hline 27 & New Hampshire & 795 & 19.1 & 27 & New Hampshire & 1,472 & 35.3 \\
\hline
\end{tabular}


Table 4. Farm typology for retirement and residential / lifestyle categories, ranked by state, 2007.

\begin{tabular}{|c|c|c|c|c|c|c|c|}
\hline \multirow[t]{2}{*}{ Rank } & \multirow[t]{2}{*}{ State } & \multicolumn{2}{|c|}{ Retirement } & \multirow[t]{2}{*}{ Rank } & \multirow[t]{2}{*}{ State } & \multicolumn{2}{|c|}{ Residential / Lifestyle } \\
\hline & & (Number) & $(\%)$ & & & (Number) & $(\%)$ \\
\hline 28 & New York & 6,795 & 18.7 & 28 & Kansas & 23,056 & 35.2 \\
\hline 29 & Pennsylvania & 11,755 & 18.6 & 29 & Florida & 16,561 & 34.9 \\
\hline 30 & Nevada & 580 & 18.5 & 30 & Wyoming & 3,824 & 34.5 \\
\hline 31 & Idaho & 4,644 & 18.3 & 31 & Nevada & 1,074 & 34.3 \\
\hline 32 & Montana & 5,312 & 18.0 & 32 & Minnesota & 27,722 & 34.2 \\
\hline 33 & Kansas & 11,690 & 17.8 & 33 & Massachusetts & 2,606 & 33.9 \\
\hline 34 & Rhode Island & 217 & 17.8 & 34 & North Carolina & 17,917 & 33.9 \\
\hline 35 & Alaska & 122 & 17.8 & 35 & Illinois & 26,001 & 33.8 \\
\hline 36 & Indiana & 10,599 & 17.4 & 36 & Maryland & 4,341 & 33.8 \\
\hline 37 & Illinois & 13,359 & 17.4 & 37 & Vermont & 2,340 & 33.5 \\
\hline 38 & Colorado & 6,389 & 17.2 & 38 & Rhode Island & 390 & 32.0 \\
\hline 39 & Massachusetts & 1,301 & 16.9 & 39 & lowa & 29.045 & 31.3 \\
\hline 40 & Maine & 1,354 & 16.6 & 40 & New York & 11,249 & 30.9 \\
\hline 41 & Vermont & 1,162 & 16.6 & 41 & Alaska & 210 & 30.6 \\
\hline 42 & Wyoming & 1,810 & 16.4 & 42 & Montana & 9,016 & 30.5 \\
\hline 43 & Wisconsin & 12,300 & 15.7 & 43 & California & 24,566 & 30.3 \\
\hline 44 & North Dakota & 5,001 & 15.6 & 44 & Hawaii & 2,240 & 29.8 \\
\hline 45 & lowa & 13,513 & 14.6 & 45 & New Mexico & 5,914 & 28.3 \\
\hline 46 & Minnesota & 11,548 & 14.3 & 46 & South Dakota & 8,565 & 27.5 \\
\hline 47 & Arizona & 2,161 & 13.8 & 47 & Nebraska & 12,927 & 27.1 \\
\hline 48 & Delaware & 331 & 13.0 & 48 & North Dakota & 8,104 & 25.3 \\
\hline 49 & South Dakota & 3,515 & 11.3 & 49 & Arizona & 3,639 & 23.3 \\
\hline 50 & Nebraska & 5,352 & 11.2 & 50 & Delaware & 551 & 21.6 \\
\hline
\end{tabular}

